\title{
RIEMANNIAN MANIFOLDS ADMITTING AN INFINITESIMAL CONFORMAL TRANSFORMATION
}

\author{
By Kentaro Yano and Sumio Sawaki
}

\section{§1. Introduction.}

Let $M$ be a differentiable connected Riemannian manifold of dimension $n$ covered by a system of coordinate neighborhoods $\left\{U ; \xi^{h}\right\}$, and let $g_{j i}$ be components of the metric tensor with respect to this coordinate system, where and in the sequel the indices $h, i, j, \cdots$ run over the range $1,2, \cdots, n$. Let $\nabla_{\imath}, K_{k j i}{ }^{h}, K_{j i}$ and $K$ be the operator of covariant differentiation with respect to Christoffel symbols $\left\{\begin{array}{l}h \\ j i\end{array}\right\}$ formed with $g_{j i}$, the curvature tensor, the Ricci tensor and the scalar curvature respectively.

An infinitesimal transformation $v^{h}$ is said to be conformal if it satisfies

$$
\mathcal{L} g_{j i}=\nabla_{j} v_{i}+\nabla_{i} v_{j}=2 \rho g_{j i},
$$

where $\mathcal{L}$ denotes the operator of Lie differentiation with respect to $v^{h}, v_{i}=g_{i h} v^{h}$ and

$$
\rho=\frac{1}{n} \nabla_{i} v^{i}
$$

If $\rho=$ constant in (1.1), the transformation is said to be homothetic and if $\rho=0$ in (1.1), the transformation is called an isometry. It is known that an infinitesimal homothetic transformation in a compact Riemannian manifold is an isometry. (Yano $[13,14]$ ).

In 1959, Nagano and one of the present authors [18] proved

THEOREM A. If $M$ is a complete Einstein manifold of dimension $n>2$ admitting an infinitesimal conformal transformation, then $M$ is isometric to a sphere in an $(n+1)$-dimensional Euclidean space.

Generalizing Theorem A, Obata [7] proved in 1962

THEOREM B. Let $M$ be a complete Riemannian manifold of dimension $n \geqq 2$. In order for $M$ to admit a non-trivial solution $\rho$ for the system of partial differential equations

$$
\nabla_{j} \nabla_{i} \rho=-c^{2} \rho g_{j i}, \quad c=\text { const. }>0,
$$

it is necessary and sufficient that $M$ be isometric to a sphere of radius $1 / c$ in the

Received January 8, 1970. 
$(n+1)$-dimensional Euclidean space.

Also in 1962, Goldberg and Kobayashi [2] proved

THEOREM C. If a compact homogeneous Riemannian manifold $M$ of dimension $n>2$ admits an infinitesimal non-homothetic conformal transformation, then $M$ is isometric to a sphere.

Generalizing Theorem C, Lichnerowicz [6] proved in 1964

Theorem D. If a compact Riemannian manifold $M$ of dimension $n>2$ with $K=$ const. and $K_{j i} K^{j i}=$ const. admits an infinitesimal non-homothetic conformal transformation, then $M$ is isometric to a sphere.

Also generalizing Theorem C, Hsiung [3] proved in 1965

THEOREM E. If a compact Riemannian manifold $M$ of dimension $n>2$ with $K=$ const. and $K_{k j i n} K^{k j i n}=$ const. admits an infinitesimal non-homothetic conformal transformation, then $M$ is isometric to a sphere.

In 1965, Obata and one of the present authors [19] proved

Theorem F. If a compact Riemannian manifold $M$ of dimension $n \geqq 2$ with $K=$ const. admits an infinitesimal non-homothetic conformal transformation $v^{h}: \mathcal{L} g_{j i}$ $=2 \rho g_{j i}, \rho \neq$ const., and if one of the following conditions is satisfied, then $M$ is isometric to a shere.

(1) The covariant vector $v_{i}=g_{i n} v^{h}$ is the gradient of a scalar function. (See also Lichnerowicz [6].)

(2) $K_{i}^{h} \rho^{i}=k \rho^{h}$, where $\rho^{2}=\rho_{j} g^{j i}$ and $\rho_{j}=\nabla_{j} \rho, k$ being a constant.

(3) $\mathcal{L} K_{j i}=\alpha g_{j i}, \alpha$ being a scalar function.

We now put

$$
G_{j i}=K_{j i}-\frac{1}{n} K g_{j i}
$$

$$
Z_{k j i}{ }^{h}=K_{k j i}{ }^{h}-\frac{1}{n(n-1)} K\left(\delta_{k}^{h} g_{j i}-\delta_{j}^{h} g_{k i}\right)
$$

The tensor $G_{j i}$ measures the deviation of $M$ from being an Einstein space and $Z_{k j i}{ }^{h}$ that from being the space of constant curvature.

It is easily seen that

$$
G_{j i} g^{j i}=0, \quad Z_{t j i}{ }^{t}=G_{j i} .
$$

In 1966, one of the present authors [15] proved

THEOREM G. If a compact and orientable Riemannian manifold $M$ of dimension $n>2$ with $K=$ const. admits an infinitesimal non-homothetic conformal transfor- 
mation $v^{h}: \mathcal{L} g_{j i}=2 \rho g_{j i}, \rho \neq$ const. such that

$$
\int_{M} G_{j i} \rho^{j} \rho^{i} d V \geqq 0,
$$

$d V$ being the volume element of $M$, then $M$ is isometric to a sphere.

TheOREM $\mathrm{H}$. If a compact Riemannian manifold $M$ of dimension $n>2$ with scalar curvature $K=$ const. admits an infinitesimal non-homothetic conformal transformation $v^{h}$ such that

$$
\mathcal{L}\left(G_{j i} G^{j i}\right)=0
$$

or

$$
\mathcal{L}\left(Z_{k j i h} Z^{k j i h}\right)=0,
$$

then $M$ is isometric to a sphere.

Theorem $\mathrm{H}$ generalizes Theorem $\mathrm{D}$ of Lichnerowicz and Theorem $\mathrm{E}$ of Hsiung.

The inequality in (1.7) can be replaced by the equality and we have in fact

THEOREM I. In order that a compact and orientable Riemannian manifold $M$ of dimension $n>2$ with scalar curvature $K=$ const. admitting an infinitesimal nonhomothetic conformal transformation $v^{h}: \mathcal{L} g_{j i}=\rho g_{j i}, \rho \neq$ const. be isometric to a sphere, it is necessary and sufficient that

$$
\int_{M} G_{j i} \rho^{j} \rho^{i} d V=0
$$

(See Goldberg [1], Obata [7, 8, 9, 10], Weber and Goldberg [12], Yano [16] and Yano and Sawaki [20]).

We now introduce the notations

$$
f=G_{j i} G^{j i}, \quad g=Z_{k j i h} Z^{k j i h} .
$$

In 1968, the present authors [20] proved

THEOREM J. If a compact Riemannian manifold $M$ of dimension $n>2$ with $K=$ const. $\neq 0$ admits an infinitesimal non-homothetic conformal transformation $v^{h}$ such that

$$
\mathcal{L}\left\{\sum_{k=0}^{l} \alpha_{k}\left(-\frac{n-1}{K}\right)^{k} \Delta^{k}(\mathcal{L} f)+\sum_{k=0}^{m} \beta_{k}\left(-\frac{n-1}{K}\right)^{k} \Delta^{k}(\mathcal{L} g)\right\}=0
$$

where $\Delta=g^{j i} \nabla_{j} \nabla_{i}, l$ and $m$ being non-negative integers and $\alpha_{k}$ and $\beta_{k}$ constants such that the sum $\sum_{k=0}^{l} \alpha_{k}$ and $\sum_{k=0}^{m} \beta_{k}$ are non-negative and not both zero, then $M$ is isometric to a sphere. 
Theorem K. Suppose that a compact Riemannian manifold $M$ of dimension $n>2$ with $K=$ const. satisfies

$$
\alpha_{0} f-\alpha_{1} \Delta f+\beta_{0} g-\beta_{1} \Delta g=\text { const., }
$$

where $\alpha_{0}, \alpha_{1}, \beta_{0}, \beta_{1}$ are non-negative constants not all zero such that if $n>6$,

$$
\frac{8 K}{n-1} \alpha_{1} \geqq(n-6) \alpha_{0} \geqq 0, \quad \frac{8 K}{n-1} \beta_{1} \geqq(n-6) \beta_{0} \geqq 0 .
$$

If $M$ admits an infinitesimal non-homothetic conformal transformation $v^{\text {: }}$ : $\mathcal{L} g_{j i}=2 \rho g_{j i}, \rho \neq$ const., then $M$ is isometric to a sphere.

THEOREM L. If a compact Riemannian manifold $M$ of dimension $n>2$ with $K=$ const. $\neq 0$ admits an infinitesimal non-homothetic conformal transformation $v^{h}$ such that

$$
\mathcal{L} \mathcal{L}\left(\alpha_{0} f+\alpha_{1} \Delta f+\beta_{0} g+\beta_{1} \Delta g\right)=0,
$$

$\alpha_{0}, \alpha_{1}, \beta_{0}, \beta_{1}$ being constants not all zero such that

$$
\frac{4(n-1)}{K} \alpha_{0} \geqq(n+6) \alpha_{1} \geqq 0, \quad \frac{4(n-1)}{K} \beta_{0} \geqq(n+6) \beta_{1} \geqq 0,
$$

then $M$ is isometric to a sphere.

We now put

$$
W_{k j i h}=a Z_{k j i h}+\frac{b}{n-1}\left(g_{k h} G_{j i}-g_{j h} G_{k i}+G_{k h} g_{j i}-G_{j h} g_{k i}\right),
$$

$a$ and $b$ being constants not both zero. It is easily seen that

$$
W_{k j i n} g^{k h}=(a+b) G_{j i}
$$

and that, when $a+b=0$,

$$
W_{k j i h}=\alpha C_{k j i h},
$$

where $C_{k j i n}$ are covariant components of the Weyl conformal curvature tensor.

The present authors [20] proved

Theorem M. Suppose that a compact Riemannian manifold $M$ of dimension $n>2$ with $K=$ const. admits an infinitesimal non-homothetic conformal transformation $v^{h}$. If

$$
\mathcal{L} \mathcal{L}\left(W_{k j i h} W^{k i i h}\right)=0,
$$

$a$ and $b$ being constants not both zero such that $a+b \neq 0, M$ is isometric to a sphere.

In 1968, Goldberg [1] proved 
TheOREM N. If a Riemannian manifold $M$ is complete, of dimension $n>2$, with $K=$ const. $>0$ and if it admits an infinitesimal non-homothetic conformal transformation $v^{h}: \mathcal{L} g_{j i}=2 g_{j i}, \rho \neq$ const., then

$$
\rho^{2} K^{2} \leqq n(n-1)^{2}\left(\nabla_{j} \rho_{i}\right)\left(\nabla^{j} \rho^{i}\right),
$$

where $\nabla^{j}=g^{j i} \nabla_{i}$, equality holding if and only if $M$ is isometric to a sphere.

In 1969, one of the present authors [16] proved

Theorem O. If $M$ is compact, orientable, of dimension $n>2$, with $K=$ const., and if it admits an infinitesimal non-homothetic conformal transformation $v^{h}: \mathcal{L} g_{j i}$ $=2 \rho g_{j i}, \rho \neq$ const., then

$$
n(n-1) \int_{M} K_{j i} \rho^{j} \rho^{i} d V \leqq K^{2} \int_{M} \rho^{2} d V
$$

equality holding if and only if $M$ is isometric to a sphere.

In 1959, Ishihara and Tashiro [5] proved

Theorem P. If a compact Riemannian manifold $M$ of dimension $n>2$ admits a non-homothetic function $\rho$ such that

$$
\nabla_{j} \nabla_{i} \rho=\frac{1}{n} \Delta \rho g_{j i}
$$

then $M$ is conformal to a sphere in an $(n+1)$-dimensional Euclidean space.

Using Theorem $\mathrm{P}$, one of the present authors [17] proved

THEOREM Q. If $M$ is a compact and orientable Riemannian manifold of dimension $n>2$ and admits an infinitesimal conformal transformation $v^{h}: \mathcal{L} g_{j i}=2 \rho g_{j i}$, $\rho \neq$ const. such that

$$
\mathcal{L} K=0
$$

and

$$
\int_{M}\left[K_{j i} \rho^{\jmath} \rho^{i}-\frac{1}{n(n-1)} \rho^{2} K^{2}\right] d V \geqq 0,
$$

then $M$ is conformal to a a sphere.

THEOREM R. If $M$ is a compact and orientable Riemannian manifold of dimension $n>2$ and admits an infinitesimal non-homothetic conformal transformation $v^{h}: \mathcal{L} g_{j i}=2 \rho g_{j i}, \rho \neq$ const. such that

$$
\left\{\begin{array}{l}
\mathcal{L} K=0, \quad \mathcal{L}\left(G_{j i} G^{j i}\right)=0, \\
\frac{1}{n-1} \int_{M} \rho^{2} K^{2} d V \leqq \int_{M} K \rho_{i} \rho^{i} d V,
\end{array}\right.
$$


or

$$
\left\{\begin{array}{l}
\mathcal{L} K=0, \quad \mathcal{L}\left(Z_{k j i n} Z^{k j i n}\right)=0 \\
\frac{1}{n-1} \int_{M} \rho^{2} K^{2} d V \leqq \int_{M} K \rho_{i} \rho^{i} d V
\end{array}\right.
$$

then $M$ is conformal to a sphere.

In 1965, Tashiro [11] proved (See also Ishihara [4])

Theorem S. If a complete Riemannian manifold $M$ admits a complete nonisometric concircular infinitesimal transformation $v^{h}$, that is, a vector field satisfying

$$
\mathcal{L} g_{j i}=2 \rho g_{j i}, \quad \nabla_{j} \nabla_{i} \rho=\frac{1}{n} \Delta \rho g_{j i}
$$

then $M$ is a locally Euclidean space or is isometric to a sphere. In the former case, the vector field is homothetic.

If we use this theorem, the conclusion in Theorem $\mathrm{Q}$ and $\mathrm{R}$ can be replaced by that the manifold is isometric to a sphere. We owe this remark to Professor Tashiro.

In almost all theorems mentioned above, one assumes that scalar curvature of the manifold is constant.

The main purpose of the present paper is to generalize some of these theorems to the case in which the scalar curvature is not necessarily constant.

We assume that the Riemannian manifold we consider is compact and orientable. If $M$ is not orientable, we have only to take an orientable double covering space of the manifold.

\section{§ 2. Preliminaries.}

Using the Ricci identity, $\nabla_{k} \nabla_{j} v^{h}-\nabla_{j} \nabla_{k} v^{h}=K_{k j i}{ }^{h} v^{2}$, which is valid for an arbitrary vector field $v^{h}$ in $M$, we can prove

$$
\begin{aligned}
& \nabla^{j}\left[\left(\nabla_{j} v_{i}+\nabla_{i} v_{\jmath}-\frac{1}{n} \nabla_{t} v^{t} g_{j i}\right) v^{i}\right] \\
= & {\left[g^{k j} \nabla_{k} \nabla_{j} v_{i}+K_{i}^{t} v_{t}+\frac{n-2}{n} \nabla_{i}\left(\nabla_{t} v^{t}\right)\right] v^{i} } \\
& +\frac{1}{2}\left(\nabla_{j} v_{i}+\nabla_{i} v_{\jmath}-\frac{2}{n} \nabla_{t} v^{t} g_{j i}\right)\left(\nabla^{j} v^{i}+\nabla^{i} v^{\jmath}-\frac{2}{n} \nabla_{s} v^{s} g^{j i}\right) .
\end{aligned}
$$

Thus assuming $M$ to be compact and orientable and integrating the above equality, we find, by Green's theorem, 


$$
\begin{aligned}
& \int_{M}\left[g^{k j} \nabla_{k} \nabla_{j} v_{i}+K_{\imath} v_{t}+\frac{n-2}{n} \nabla_{i}\left(\nabla_{t} v^{t}\right)\right] v^{i} d V \\
& +\frac{1}{2} \int_{M}\left(\nabla_{j} v_{i}+\nabla_{i} v_{j}-\frac{2}{n} \nabla_{t} v^{t} g_{j i}\right)\left(\nabla^{j} v^{i}+\nabla^{i} v^{j}-\frac{2}{n} \nabla_{s} v^{s} g^{j i}\right) d V=0 .
\end{aligned}
$$

In particular, when $v_{i}$ is a gradient vector field, that is, when there exists a scalar function $\rho$ such that $v_{i}=\rho_{i}=\nabla_{i} \rho$, we have from (2.1)

$$
\begin{gathered}
\int_{M}\left(g^{k j} \nabla_{k} \nabla_{j} \rho_{i}+K_{i}^{t} \rho_{t}+\frac{n-2}{n} \nabla_{i} \Delta \rho\right) \rho^{i} d V \\
+2 \int_{M}\left(\nabla_{j} \rho_{i}-\frac{1}{n} \Delta \rho g_{j i}\right)\left(\nabla^{j} \rho^{i}-\frac{1}{n} \Delta \rho g^{j i}\right) d V=0 .
\end{gathered}
$$

Using the identity

$$
g^{k j} \nabla_{k} \nabla_{j} \rho_{i}=K_{i}^{t} \rho_{t}+\nabla_{i} \Delta \rho,
$$

we find from (2.2)

$$
\begin{aligned}
& \int_{M}\left(K_{j i} \rho^{j} \rho^{i}+\frac{n-1}{n} \rho^{i} \nabla_{i} \Delta \rho\right) d V \\
+ & \int_{M}\left(\nabla_{j} \rho_{i}-\frac{1}{n} \Delta \rho g_{j i}\right)\left(\nabla^{j} \rho^{i}-\frac{1}{n} \Delta \rho g^{j i}\right) d V=0
\end{aligned}
$$

or using Green's theorem

$$
\begin{aligned}
& \int_{M}\left[K_{j i} \rho^{j} \rho^{i}-\frac{n-1}{n}(\Delta \rho)^{2}\right] d V \\
+ & \int_{M}\left(\nabla_{j} \rho_{i}-\frac{1}{n} \Delta \rho g_{j i}\right)\left(\nabla^{j} \rho^{i}-\frac{1}{n} \Delta \rho g^{j i}\right) d V=0,
\end{aligned}
$$

which is valid for an arbitray scalar function $\rho$.

Now for an infinitesimal conformal transformation $v^{h}$ in $M$, we have

$$
\mathcal{L} g_{j i}=\nabla_{j} v_{i}+\nabla_{i} v_{j}=2 \rho g_{i j}
$$

from which we can deduce

$$
g^{k j} \nabla_{k} \nabla_{j} v^{h}+K_{i}^{h} v^{i}+\frac{n-2}{n} \nabla^{h}\left(\nabla_{t} v^{t}\right)=0
$$

If a vector field $v^{h}$ in a compact and orientable Riemannian manifold satisfies (2.6), then we can conclude from (2.1)

$$
\nabla_{j} v_{i}+\nabla_{i} v_{j}-\frac{2}{n} \nabla_{t} v^{t} g_{j i}=0
$$


which shows that (2.6) is a necessary and sufficient condition for $v^{h}$ to be an infinitesimal conformal transformation in a compact Riemannian manifold $M$. (See, for example, Yano [14]).

We shall need in the sequel the formula

$$
\Delta(\mathcal{L} F)-\mathcal{L}(\Delta F)=2 \rho \Delta F-(n-2) \rho^{i} V_{i} F,
$$

which is valid for an arbitrary function $F$ and for an infinitesimal conformal transformation $v^{h}$. The formula will be proved by using (2.6) and

$$
g^{j i} \nabla_{j} \nabla_{i} \nabla_{h} F-K_{h}^{i} \nabla_{i} F=\nabla_{h}(\Delta F) .
$$

From (2.7), we have for $F=K$

$$
\rho[\Delta(\mathcal{L} K)-\mathcal{L}(\Delta K)]=2 \rho^{2} \Delta K-\frac{n-2}{2}\left(\nabla^{i} \rho^{2}\right) \nabla_{i} K,
$$

from which

$$
\begin{aligned}
\int_{M} \rho[\Delta(\mathcal{L} K)-\mathcal{L}(\Delta K)] d V & =\int_{M}\left[2 \rho^{2} \Delta K-\frac{n-2}{2}\left(\nabla^{i} \rho^{2}\right) \nabla_{i} K\right] d V \\
& =\int_{M}\left[2 \rho^{2} \Delta K+\frac{n-2}{2} \rho^{2} \Delta K\right] d V \\
& =\frac{n+2}{2} \int_{M} \rho^{2} \Delta K d V
\end{aligned}
$$

by virtue of Green's theorem, or

$$
\int_{M} \rho^{2} \Delta K d V=\frac{2}{n+2} \int_{M} \rho[\Delta(\mathcal{L} K)-\mathcal{L}(\Delta K)] d V
$$

From (2.5), we find

$$
\begin{gathered}
\mathcal{L} K_{k j i}^{h}=-\delta_{k}^{h} \nabla_{j} \rho_{i}+\delta_{j}^{h} \nabla_{k} \rho_{i}-\nabla_{k} \rho^{h} g_{j i}+\nabla_{j} \rho^{h} g_{k i}, \\
\mathcal{L} K_{j i}=-(n-2) \nabla_{j} \rho_{i}-\Delta \rho g_{j i}, \\
\mathcal{L} K=-2(n-1) \Delta \rho-2 \rho K .
\end{gathered}
$$
tives:

These formulas will be proved by applying well known formulas on Lie deriva-

$$
\mathcal{L}\left\{\begin{array}{l}
h \\
j i
\end{array}\right\}=\frac{1}{2} g^{h t}\left(\nabla_{j} \mathcal{L} g_{i t}+\nabla_{i} \mathcal{L} g_{j t}-\nabla_{t} \mathcal{L} g_{j i}\right)
$$

and

$$
\mathcal{L} K_{k j i}{ }^{h}=\nabla_{k} \mathcal{L}\left\{\begin{array}{l}
h \\
j i
\end{array}\right\}-\nabla_{j} \mathcal{L}\left\{\begin{array}{c}
h \\
k i
\end{array}\right\} \cdot
$$


(See, for example, Yano [14]).

From (2.10), (2.11) and (2.12), we find

$$
\mathcal{L} G_{j i}=-(n-2)\left(\nabla_{j} \rho_{i}-\frac{1}{n} \Delta \rho g_{j i}\right)
$$

and

$$
\mathcal{L} Z_{k j i}{ }^{h}=-\delta_{k}^{h} \nabla_{j} \rho_{i}+\delta_{j}^{h} \nabla_{k} \rho_{i}-\nabla_{k} \rho^{h} g_{j i}+\nabla_{j} \rho^{h} g_{k i}+\frac{2}{n} \Delta \rho\left(\delta_{k}^{h} g_{j i}-\delta_{j}^{h} g_{k i}\right) .
$$

Using (2.13) and $\mathcal{L} g^{j i}=-2 \rho g^{j i}$, we have

$$
\begin{aligned}
\mathcal{L}\left(G_{j i} G^{j i}\right) & =2\left(\mathcal{L} G_{j i}\right) G^{j i}-4 G_{j i} G^{j i} \rho^{2} \\
& =-2(n-2)\left(\nabla_{j} \rho_{i}-\frac{1}{n} \Delta \rho g_{j i}\right) G^{j i}-4 G_{j i} G^{j i} \rho,
\end{aligned}
$$

or

$$
G_{j i} \nabla^{j} \rho^{\imath}=-\frac{2}{n-2} G_{j i} G^{j i} \rho-\frac{1}{2(n-2)} \mathcal{L}\left(G_{j i} G^{j i}\right),
$$

by virtue of $G_{j i} g^{j i}=0$.

On the other hand, we have

$$
\nabla^{j}\left(G_{j i} \rho \rho^{i}\right)=\left(\nabla^{j} G_{j i}\right) \rho \rho^{2}+G_{j i} \rho^{j} \rho^{i}+\rho G_{j i} \nabla^{j} \rho^{i},
$$

from which, taking account of (2.15) and

$$
\nabla^{\jmath} G_{j i}=\frac{n-2}{2 n} \nabla_{i} K
$$

we find

$$
\nabla^{j}\left(G_{j i} \rho \rho^{i}\right)=\frac{n-2}{2 n} \rho \rho^{i} \nabla_{i} K+G_{j i} \rho^{j} \rho^{i}-\frac{2}{n-2} \rho^{2} G_{j i} G^{j i}-\frac{1}{2(n-2)} \rho \mathcal{L}\left(G_{j i} G^{j i}\right) .
$$

Integrating this over $M$, we obtain

$$
0=\int_{M}\left[\frac{n-2}{2 n} \rho \rho^{i} \nabla_{i} K+G_{j i} \rho^{j} \rho^{2}-\frac{2}{n-2} \rho^{2} G_{j i} G^{j i}-\frac{1}{2(n-2)} \rho \mathcal{L}\left(G_{j i} G^{j i}\right)\right] d V,
$$

or

(2.17) $\int_{M} G_{j i} \rho^{\jmath} \rho^{\imath} d V=\frac{1}{n-2} \int_{M}\left[2 \rho^{2} G_{j i} G^{j i}+\frac{1}{2} \rho \mathcal{L}\left(G_{j i} G^{j i}\right)+\frac{(n-2)^{2}}{4 n} \rho^{2} \Delta K\right] d V$,

(2.18) $\int_{M} G_{j i} \rho^{\jmath} \rho^{2} d V=\frac{1}{n-2} \int_{M}\left[2 \rho^{2} G_{j i} G^{j i}+\frac{1}{2} \rho \mathcal{L}\left(G_{j i} G^{j i}\right)\right.$

$$
\left.+\frac{(n-2)^{2}}{2 n(n+2)} \rho(\Delta \mathcal{L} K-\mathcal{L} \Delta K)\right] d V
$$


by virtue of (2.9).

Taking account of (2.14), we have

$$
\begin{aligned}
\mathcal{L}\left(Z_{k j i n} Z^{k j i h}\right)= & 2\left(\mathcal{L} Z_{k j i}^{h}\right) Z^{k j i}{ }_{h}-4 Z_{k j i n} Z^{k j i \hbar} \rho \\
= & 2\left[-\delta_{k}^{h} \nabla_{j} \rho_{i}+\delta_{j}^{h} \nabla_{k} \rho_{i}-\nabla_{k} \rho^{h} g_{j i}+\nabla_{j} \rho^{h} g_{k i}\right. \\
& \left.+\frac{2}{n} \Delta \rho\left(\delta_{k}^{h} g_{j i}-\delta_{j}^{h} g_{k i}\right)\right] Z^{k j i}-4 Z_{k j i h} Z^{k j i \hbar} \rho,
\end{aligned}
$$

or

$$
G_{j i} \nabla^{j} \rho^{i}=-\frac{1}{2} Z_{k j i n} Z^{k j i \hbar} \rho-\frac{1}{8} \mathcal{L}\left(Z_{k j i n} Z^{k j i h}\right),
$$

by virtue of $G_{j i} g^{j i}=0$ and $Z_{k j i n} g^{k h}=G_{j i}$.

On the other hand, we have

$$
\begin{aligned}
\nabla^{j}\left(G_{j i} \rho \rho^{i}\right) & =\left(\nabla^{j} G_{j i}\right) \rho \rho^{2}+G_{j i} \rho^{\jmath} \rho^{2}+\rho G_{j i} \nabla^{\jmath} \rho^{2} \\
& =\frac{n-2}{2 n} \rho \rho^{i} \nabla_{i} K+G_{j i} \rho^{\jmath} \rho^{2}-\frac{1}{2} \rho^{2} Z_{k j i n} Z^{k j i h}-\frac{1}{8} \rho \mathcal{L}\left(Z_{k j i n} Z^{k j i n}\right),
\end{aligned}
$$

by virtue of (2.16) and (2.19), from which

$$
0=\int_{M}\left[\frac{n-2}{2 n} \rho \rho^{i} \nabla_{i} K+G_{j i} \rho^{\jmath} \rho^{2}-\frac{1}{2} \rho^{2} Z_{k j i n} Z^{k j i h}-\frac{1}{8} \rho \mathcal{L}\left(Z_{k j i n} Z^{k j i n}\right)\right] d V,
$$

or

(2. 20) $\int_{M} G_{j i} \rho^{\jmath} \rho^{i} d V=\int_{M}\left[\frac{1}{2} \rho^{2} Z_{k j i h} Z^{k j i n}+\frac{1}{8} \rho \mathcal{L}\left(Z_{k j i h} Z^{k j i n}\right)+\frac{n-2}{4 n} \rho^{2} \Delta K\right] d V$,

or

(2. 21) $\quad \int_{M} G_{j i} \rho^{\jmath} \rho^{2} d V=\int_{M}\left[\frac{1}{2} \rho^{2} Z_{k j i h} Z^{k j i n}+\frac{1}{8} \rho \mathcal{L}\left(Z_{k j i n} Z^{k j i n}\right)\right.$

$$
\left.+\frac{n-2}{2 n(n+2)} \rho(\Delta \mathcal{L} K-\mathcal{L} \Delta K)\right] d V
$$

by virtue of (2.9).

For the Lie derivative $\mathcal{L} W_{k j i n}$ of $W_{k j i n}$ defined by (1.17), we have

$$
\mathcal{L} W_{k j i h}=2 a \rho Z_{k j i n}+\frac{2 b \rho}{n-2}\left(g_{k h} G_{j i}-g_{j h} G_{k i}+G_{k h} g_{j i}-G_{j h} g_{k i}\right)
$$

$$
-(a+b)\left[\left(g_{k h} \nabla_{j} \rho_{i}-g_{j h} \nabla_{k} \rho_{i}+\nabla_{k} \rho_{h} g_{j i}-\nabla_{j} \rho_{h} g_{k i}\right)-\frac{2}{n} \Delta \rho\left(g_{k h} g_{j i}-g_{j h} g_{k i}\right)\right],
$$


by virtue of (2.13), (2.14) and

$$
\begin{aligned}
\mathcal{L} Z_{k j i h} & =\mathcal{L}\left(Z_{k j i}{ }^{t} g_{t h}\right) \\
& =\left(\mathcal{L} Z_{k j i}{ }^{t}\right) g_{t h}+2 \rho Z_{k j i h} .
\end{aligned}
$$

Using (2.22), we have

$$
\mathcal{L}\left(W_{k j i h} W^{k j i n}\right)=-4 \rho W_{k j i h} W^{k j i n}-8(a+b)^{2} G_{j i} \nabla^{j} \rho^{i},
$$

or

$$
8(a+b)^{2} G_{j i} \nabla^{j} \rho^{i}=-4 \rho W_{k j i n} W^{k j i h}-\mathcal{L}\left(W_{k j i h} W^{k j i h}\right) .
$$

On the other hand, we have

$$
8(a+b)^{2} \nabla^{j}\left(G_{j i} \rho \rho^{i}\right)=8(a+b)^{2}\left(\nabla^{j} G_{j i}\right) \rho \rho^{i}+8(a+b)^{2} G_{j i} \rho^{j} \rho^{i}+8(a+b)^{2} \rho G_{j i} \nabla^{j} \rho^{i},
$$

and consequently

$$
\begin{aligned}
8(a+b)^{2} \nabla^{j}\left(G_{j i} \rho \rho^{i}\right)= & \frac{4(n-2)}{n}(a+b)^{2} \rho \rho^{i} V_{i} K \\
& +8(a+b)^{2} G_{j i} \rho^{j} \rho^{i}-4 \rho^{2} W_{k j i h} W^{k j i h}-\rho \mathcal{L}\left(W_{k j i h} W^{k j i h}\right),
\end{aligned}
$$

by virtue of (2.16) and (2.23), from which

$$
0=\int_{M}\left[\frac{4(n-2)}{n}(a+b)^{2} \rho \rho^{i} \nabla_{i} K+8(a+b)^{2} G_{j i} \rho^{j} \rho^{i}-4 \rho^{2} W_{k j i h} W^{k j i n}-\rho \mathcal{L}\left(W_{k j i h} W^{k j i n}\right)\right] d V,
$$

or

$$
\begin{aligned}
& 8(a+b)^{2} \int_{M} G_{j i} \rho^{j} \rho^{i} d V \\
= & \int_{M}\left[4 \rho^{2} W_{k j i n} W^{k j i h}+\rho \mathcal{L}\left(W_{k j i h} W^{k j i n}\right)+\frac{2(n-2)}{n}(a+b)^{2} \rho^{2} \Delta K\right] d V,
\end{aligned}
$$

or

$$
\begin{aligned}
& 8(a+b)^{2} \int_{M} G_{j i} \rho^{j} \rho^{i} d V \\
= & \int_{M}\left[4 \rho^{2} W_{k j i h} W^{k j i n}+\rho \mathcal{L}\left(W_{k j i n} W^{k j i h}\right)+\frac{4(n-2)}{n(n+2)}(a+b)^{2} \rho(\Delta \mathcal{L} K-\mathcal{L} \Delta K)\right] d V,
\end{aligned}
$$

by virtue of (2.9), or

$$
\int_{M} G_{j i} \rho^{j} \rho^{i} d V=\frac{1}{2(a+b)^{2}} \int_{M} \rho^{2} W_{k j i h} W^{k j i n} d V+\frac{1}{8(a+b)^{2}} \int_{M} \rho \mathcal{L}\left(W_{k j i h} W^{k j i h}\right) d V
$$

$$
+\frac{n-2}{2 n(n+2)} \int_{M} \rho(\Delta \mathcal{L} K-\mathcal{L} \Delta K) d V \text {. }
$$




\section{§3. A generalization of Theorem $\mathrm{H}$.}

For an infinitesimal conformal transformation $v^{h}$, we have from (2.12)

$$
\Delta \rho=-\frac{1}{n-1} \rho K-\frac{1}{2(n-1)} \mathcal{L} K .
$$

Substituting this into (2.3), we find

$$
\begin{aligned}
\int_{M}\left[K_{j i} \rho^{j} \rho^{i}-\frac{n-1}{n}\right. & \left.\rho^{i} \nabla_{i}\left(\frac{1}{n-1} \rho K+\frac{1}{2(n-1)} \mathcal{L} K\right)\right] d V \\
& +\int_{M}\left(\nabla_{j} \rho_{i}-\frac{1}{n} \Delta \rho g_{j i}\right)\left(\nabla^{j} \rho^{i}-\frac{1}{n} \Delta \rho g^{j i}\right) d V=0,
\end{aligned}
$$

from which

$$
\begin{aligned}
\int_{M}\left[G_{j i} \rho^{j} \rho^{i}+\frac{1}{2 n} \rho^{2} \Delta K\right. & \left.+\frac{1}{2 n} \rho \Delta(\mathcal{L} K)\right] d V \\
& +\int_{M}\left(\nabla_{j} \rho_{i}-\frac{1}{n} \Delta \rho g_{j i}\right)\left(\nabla^{j} \rho^{i}-\frac{1}{n} \Delta \rho g^{j i}\right) d V=0 .
\end{aligned}
$$

Substituting (2.9) into (3.2), we find

$$
\begin{aligned}
\int_{M}\left[G_{j i} \rho^{\jmath} \rho^{i}\right. & \left.+\frac{1}{n(n+2)} \rho(\Delta \mathcal{L} K-\mathcal{L} \Delta K)+\frac{1}{2 n} \Delta(\mathcal{L} K)\right] d V \\
& +\int_{M}\left(\nabla_{j} \rho_{i}-\frac{1}{n} \Delta \rho g_{j i}\right)\left(\nabla^{j} \rho^{i}-\frac{1}{n} \Delta \rho g^{j i}\right) d V=0,
\end{aligned}
$$

and consequently

$$
\begin{aligned}
\int_{M}\left[G_{j i} \rho^{j} \rho^{i}\right. & \left.+\frac{n+4}{2 n(n+2)} \rho \Delta(\mathcal{L} K)-\frac{1}{n(n+2)} \rho \mathcal{L}(\Delta K)\right] d V \\
& +\int_{M}\left(\nabla_{j} \rho_{i}-\frac{1}{n} \Delta \rho g_{j i}\right)\left(\nabla^{j} \rho^{i}-\frac{1}{n} \Delta \rho g^{j i}\right) d V=0 .
\end{aligned}
$$

Substituting (2.18) into (3.3), we find

$$
\begin{aligned}
& \int_{M}\left[\frac{2}{n-2} \rho^{2} G_{j i} G^{j i}+\frac{1}{2(n-2)} \rho \mathcal{L}\left(G_{j i} G^{j i}\right)\right. \\
& \left.\quad+\frac{n-2}{2 n(n+2)} \rho(\Delta \mathcal{L} K-\mathcal{L} \Delta K)+\frac{n+4}{2 n(n+2)} \rho \Delta(\mathcal{L} K)-\frac{1}{n(n+2)} \rho \mathcal{L} \Delta K\right] d V \\
& \quad+\int_{M}\left(\nabla_{j} \rho_{i}-\frac{1}{n} \Delta \rho g_{j i}\right)\left(\nabla^{j} \rho^{i}-\frac{1}{n} \Delta \rho g^{j i}\right) d V=0,
\end{aligned}
$$


or

$$
\begin{aligned}
\int_{M}\left[4 G_{j i} G^{j i} \rho^{2}\right. & \left.+\rho\left\{\mathcal{L}\left(G_{j i} G^{j i}\right)+\frac{2(n+1)(n-2)}{n(n+2)} \Delta \mathcal{L} K-\frac{n-2}{n+2} \mathcal{L} \Delta K\right\}\right] d V \\
& +2(n-2) \int_{M}\left(\nabla_{j} \rho_{i}-\frac{1}{n} \Delta \rho g_{j i}\right)\left(\nabla^{j} \rho^{i}-\frac{1}{n} \Delta \rho g^{j i}\right) d V=0
\end{aligned}
$$

or

$$
\begin{gathered}
\int_{M}\left[4 G_{j i} G^{j i} \rho^{2}-\frac{1}{n} \mathcal{L}\left\{\mathcal{L}\left(G_{j i} G^{j i}\right)+\frac{2(n+1)(n-2)}{n(n+2)} \Delta \mathcal{L} K-\frac{n-2}{n+2} \mathcal{L} \Delta K\right\}\right] d V \\
+2(n-2) \int_{M}\left(\nabla_{j} \rho_{i}-\frac{1}{n} \Delta \rho g_{j i}\right)\left(\nabla^{j} \rho^{i}-\frac{1}{n} \Delta \rho g^{j i}\right) d V=0
\end{gathered}
$$

where we have used

$$
\int_{M} \rho F d V=\int_{M} \frac{1}{n}\left(\nabla_{i} v^{i}\right) F d V=-\frac{1}{n} \int_{M} \mathcal{L} F d V
$$

for an arbitrary function $F$.

Thus, applying Theorem S of Tashiro, we have

Theorem 3.1. If a compact Riemannian manifold $M$ of dimension $n>2$ admits an infinitesimal non-homothetic conformal transformation $v^{h}$ such that

$$
\mathcal{L}\left[\mathcal{L}\left(G_{j i} G^{j i}-\frac{n-2}{n+2} \Delta K\right)+\frac{2(n+1)(n-2)}{n(n+2)} \Delta \mathcal{L} K\right]=0,
$$

then $M$ is isometric to a sphere.

CoRollary 1. If a compact Riemannian manifold $M$ of dimension $n>2$ admits an infinitesimal non-homothetic conformal transformation $v^{h}$ such that one of the following conditions is satisfied:

$$
\begin{gathered}
\mathcal{L}\left(G_{j i} G^{j i}-\frac{n-2}{n+2} \Delta K\right)+\frac{2(n+1)(n-2)}{n(n+2)} \Delta \mathcal{L} K=\text { const. } \\
\mathcal{L} K=0, \quad \mathcal{L} \mathcal{L}\left(G_{j i} G^{j i}-\frac{n-2}{n+2} \Delta K\right)=0 \\
\mathcal{L} K=0, \quad \mathcal{L}\left(G_{j i} G^{j i}-\frac{n-2}{n+2} \Delta K\right)=0 \\
\mathcal{L} K=0, \quad G_{j i} G^{j i}-\frac{n-2}{n+2} \Delta K=\text { const. }
\end{gathered}
$$

then $M$ is isometric to a sphere. 
Corollary 2. If a compact Riemannian manifold $M$ of dimension $n>2$ and with constant scalar curvature $K$ admits an infinitesimal non-homothetic conformal transformation $v^{h}$ such that one of the following conditions is satisfied:

$$
\mathcal{L} \mathcal{L}\left(G_{j i} G^{j i}\right)=0,
$$

$$
\mathcal{L}\left(G_{j i} G^{j i}\right)=0,
$$

$$
G_{j i} G^{j i}=\text { const., }
$$

then $M$ is isometric to a sphere.

Now substituting (2.21) into (3.3), we find

$$
\begin{aligned}
\int_{M}\left[\frac{1}{2} \rho^{2} Z_{k j i n} Z^{k j i n}\right. & +\frac{1}{8} \rho \mathcal{L}\left(Z_{k j i n} Z^{k j i n}\right)+\frac{n-2}{2 n(n+2)} \rho(\Delta \mathcal{L} K-\mathcal{L} \Delta K)+\frac{n+4}{2 n(n+2)} \rho \Delta \mathcal{L} K \\
& \left.-\frac{1}{n(n+2)} \rho \mathcal{L} \Delta K\right] d V+\int_{M}\left(\nabla_{j} \rho_{i}-\frac{1}{n} \Delta \rho g_{j i}\right)\left(\nabla^{j} \rho^{i}-\frac{1}{n} \Delta \rho g^{j i}\right) d V=0
\end{aligned}
$$

or

$$
\int_{M}\left[\frac{1}{2} \rho^{2} Z_{k j i n} Z^{k j i n}+\frac{1}{8} \rho\left\{\mathcal{L}\left(Z_{k j i n} Z^{k j i n}\right)+\frac{8(n+1)}{n(n+2)} \Delta \mathcal{L} K-\frac{4}{n+2} \mathcal{L} \Delta K\right\}\right] d V
$$

$$
+\int_{M}\left(\nabla_{j} \rho_{i}-\frac{1}{n} \Delta \rho g_{j i}\right)\left(\nabla^{\jmath} \rho^{2}-\frac{1}{n} \Delta \rho g^{j i}\right) d V=0
$$

or

$$
\begin{aligned}
\int_{M}\left[\frac{1}{2} \rho^{2} Z_{k j i n} Z^{k j i n}\right. & \left.-\frac{1}{8 n} \mathcal{L}\left\{\mathcal{L}\left(Z_{k j i n} Z^{k j i n}\right)+\frac{8(n+1)}{n(n+2)} \Delta \mathcal{L} K-\frac{4}{n+2} \mathcal{L} \Delta K\right\}\right] d V \\
& +\int_{M}\left(\nabla_{j} \rho_{i}-\frac{1}{n} \Delta \rho g_{j i}\right)\left(\nabla^{j} \rho^{i}-\frac{1}{n} \Delta \rho g^{j i}\right) d V=0 .
\end{aligned}
$$

Thus, applying Theorem S of Tashiro, we have

THEOREM 3.2. If a compact Riemannian manifold $M$ of dimension $n>2$ admits an infinitesimal non-homothetic conformal transformation $v^{h}$ such that

$$
\mathcal{L}\left[\mathcal{L}\left(Z_{k j i h} Z^{k j i n}-\frac{4}{n+2} \Delta K\right)+\frac{8(n+1)}{n(n+2)} \Delta \mathcal{L} K\right]=0
$$

then $M$ is isometric to a sphere.

Corollary 1. If a compact Riemannian manifold $M$ of dimension $n>2$ admits an infinitesimal non-homothetic conformal transformation $v^{h}$ such that one of the following conditions is satisfied: 


$$
\mathcal{L}\left(Z_{k j i h} Z^{k j i n}-\frac{4}{n+2} \Delta K\right)+\frac{8(n+1)}{n(n+2)} \Delta \mathcal{L} K=\text { const. }
$$

$$
\mathcal{L} K=0, \quad \mathcal{L} \mathcal{L}\left(Z_{k j i n} Z^{k j i n}-\frac{4}{n+2} \Delta K\right)=0,
$$

$$
\mathcal{L} K=0, \quad \mathcal{L}\left(Z_{k j i n} Z^{k j i n}-\frac{4}{n+2} \Delta K\right)=0,
$$

$$
\mathcal{L} K=0, \quad Z_{k j i h} Z^{k j i h}-\frac{4}{n+2} \Delta K=\text { const. }
$$

then $M$ is isometric to a sphere.

Corollary 2. If a compact Riemannian manifold $M$ of dimension $n>2$ and with constant scalar curvature $K$ admits an infinitesimal non-homothetic conformal transformation $v^{h}$ such that one of the following conditions is satisfied:

$$
\begin{aligned}
& \mathcal{L} \mathcal{L}\left(Z_{k j i h} Z^{k j i h}\right)=0, \\
& \mathcal{L}\left(Z_{k j i h} Z^{k j i h}\right)=0, \\
& Z_{k j i h} Z^{k j i n}=\text { const., }
\end{aligned}
$$

then $M$ is isometric to a sphere.

\section{§4. A generalization of Theorem $\mathrm{M}$.}

Substituting (2.25) into (3.3), we find

$$
\begin{aligned}
& \int_{M}\left[\frac{1}{2(a+b)^{2}} \rho^{2} W_{k j i h} W^{k j i n}+\frac{1}{8(a+b)^{2}} \rho \mathcal{L}\left(W_{k j i n} W^{k j i n}\right)\right. \\
& \left.\quad+\frac{n-2}{2 n(n+2)} \rho(\Delta \mathcal{L} K-\mathcal{L} \Delta K)+\frac{n+4}{2 n(n+2)} \rho \Delta(\mathcal{L} K)-\frac{1}{n(n+2)} \rho \mathcal{L}(\Delta K)\right] d V \\
& \quad+\int_{M}\left(\nabla_{j} \rho_{i}-\frac{1}{n} \Delta \rho g_{j i}\right)\left(\nabla^{j} \rho^{i}-\frac{1}{n} \Delta \rho g^{j i}\right) d V=0,
\end{aligned}
$$

or

$$
\begin{gathered}
\int_{M}\left[\frac{1}{2(a+b)^{2}} \rho^{2} W_{k j i h} W^{k j i n}+\frac{1}{8(a+b)^{2}} \rho \mathcal{L}\left(W_{k j i n} W^{k j i n}\right)+\frac{n+1}{n(n+2)} \rho \Delta(\mathcal{L} K)\right. \\
\left.-\frac{1}{2(n+2)} \rho \mathcal{L}(\Delta K)\right] d V+\int_{M}\left(\nabla_{j} \rho_{i}-\frac{1}{n} \Delta \rho g_{j i}\right)\left(\nabla^{j} \rho^{i}-\frac{1}{n} \Delta \rho g^{j i}\right) d V=0,
\end{gathered}
$$


or

$$
\int_{M}\left[\frac{1}{2(a+b)^{2}} \rho^{2} W_{k j i h} W^{k j i n}-\frac{1}{n} \mathcal{L}\left\{\frac{1}{8(a+b)^{2}} \mathcal{L}\left(W_{k j i n} W^{k j i n}\right)+\frac{n+1}{n(n+2)} \Delta(\mathcal{L} K)\right.\right.
$$

$$
\left.\left.-\frac{1}{2(n+2)} \mathcal{L}(\Delta K)\right\}\right] d V+\int_{M}\left(\nabla_{j} \rho_{i}-\frac{1}{n} \Delta \rho g_{j i}\right)\left(\nabla^{j} \rho^{i}-\frac{1}{n} \Delta \rho g^{j i}\right) d V=0 .
$$

Thus, applying Theorem S of Tashiro, we obtain

THEOREM 4.1. If a compact Riemannian manifold $M$ of dimension $n>2$ admits an infinitesimal non-homothetic conformal transformation $v^{h}$ such that

$$
\mathcal{L}\left[\mathcal{L}\left(\frac{1}{8(a+b)^{2}} W_{k j i n} W^{k j i n}-\frac{1}{2(n+2)} \Delta K\right)+\frac{n+1}{n(n+2)} \Delta \mathcal{L} K\right]=0
$$

then $M$ is isometric to a sphere.

Corollary 1. If a compact Riemannian manifold $M$ of dimension $n>2$ admits an infinitesimal non-homothetic conformal transformation $v^{h}$ such that one of the following conditions is satisfied:

$$
\begin{gathered}
\mathcal{L}\left(\frac{1}{8(a+b)^{2}} W_{k j i h} W^{k j i h}-\frac{1}{2(n+2)} \Delta K\right)+\frac{n+1}{n(n+2)} \Delta \mathcal{L} K=\text { const. } \\
\mathcal{L} K=0, \quad \mathcal{L} \mathcal{L}\left(W_{k j i h} W^{k j i h}-\frac{4(a+b)^{2}}{n+2} \Delta K\right)=0 \\
\mathcal{L} K=0, \quad \mathcal{L}\left(W_{k j i h} W^{k j i n}-\frac{4(a+b)^{2}}{n+2} \Delta K\right)=0, \\
\mathcal{L} K=0, \quad W_{k j i h} W^{k j i h}-\frac{4(a+b)^{2}}{n+2} \Delta K=\text { const. },
\end{gathered}
$$

then $M$ is isometric to a sphere.

Corollary 2. If a compact Riemannian manifold $M$ of dimension $n>2$ and with constant scalar curvature $K$ admits an infinitesimal non-homothetic conformal transformation $v^{h}$ such that one of the following conditions is satisfied:

$$
\begin{aligned}
& \mathcal{L} \mathcal{L}\left(W_{k j i h} W^{k j i h}\right)=0, \\
& \mathcal{L}\left(W_{k j i h} W^{k j i h}\right)=0, \\
& W_{k j i h} W^{k j i h}=\text { const. }
\end{aligned}
$$

then $M$ is isometric to a sphere. 
§5. Generalizations of Theorem F, (2), (3).

We prove

THEOREM 5.1. Let $M$ be a compact Riemannian manifold of dimension $n \geqq 2$ and admit an infinitesimal non-homothetic conformal transformation $v^{h}$ such that

$$
\mathcal{L} K=0 \text {, }
$$

and

$$
K_{i}^{h} \rho^{i}=k \rho^{h},
$$

$k$ being a constant such that

$$
n^{2} k^{2} \geqq K^{2},
$$

then $M$ is isometric to a sphere.

Proof. We have

$$
\begin{aligned}
\nabla^{j}\left(K_{j i} \rho v^{i}\right) & =\frac{1}{2} \rho v^{i} \nabla_{i} K+K_{j i} \rho^{j} v^{i}+\rho K_{j i} \nabla^{j} v^{i} \\
& =k \rho_{i} v^{i}+\rho^{2} K \\
& =k \nabla_{i}\left(\rho v^{i}\right)-n k \rho^{2}+\rho^{2} K
\end{aligned}
$$

by virtue of

$$
\nabla^{j} K_{j i}=\frac{1}{2} \nabla_{i} K, \quad \nabla^{j} v^{i}+\nabla^{i} v^{j}=2 \rho g^{j i},
$$

and

$$
K_{j i} \rho^{j}=k \rho_{i}, \quad \nabla_{i} v^{i}=n \rho .
$$

Thus, by Green's theorem,

$$
\int_{M} \rho^{2} K d V=n k \int_{M} \rho^{2} d V
$$

Substituting

$$
\Delta \rho=-\frac{1}{n-1} \rho K
$$

obtained from (2.12) and $\mathcal{L} K=0$, into the above equation, we find

$$
-(n-1) \int_{M} \rho \Delta \rho d V=n k \int_{M} \rho^{2} d V
$$

$$
(n-1) \int_{M} \rho_{i} \rho^{i} d V=n k \int_{M} \rho^{2} d V
$$


Now, from (2.4), we have

$$
\int_{M}\left[K_{j i} \rho^{j} \rho^{2}-\frac{1}{n(n-1)} K^{2} \rho^{2}\right] d V+\int_{M}\left(\nabla_{j} \rho_{i}-\frac{1}{n} \Delta \rho g_{j i}\right)\left(\nabla^{j} \rho^{2}-\frac{1}{n} \Delta \rho g^{j i}\right) d V=0
$$

from which

$$
\int_{M}\left[k \rho_{i} \rho^{i}-\frac{1}{n(n-1)} K^{2} \rho^{2}\right] d V+\int_{M}\left(\nabla_{j} \rho_{i}-\frac{1}{n} \Delta \rho g_{j i}\right)\left(\nabla^{j} \rho^{2}-\frac{1}{n} \Delta \rho g^{j i}\right) d V=0 .
$$

Substituting (5.1) into this equation, we find

$$
\int_{M}\left[\frac{n k^{2}}{n-1} \rho^{2}-\frac{1}{n(n-1)} K^{2} \rho^{2}\right] d V+\int_{M}\left(\nabla_{j} \rho_{i}-\frac{1}{n} \Delta \rho g_{j i}\right)\left(\nabla^{j} \rho^{2}-\frac{1}{n} \Delta \rho g^{j i}\right) d V=0
$$

or

$$
\frac{1}{n(n-1)} \int_{M}\left(n^{2} k^{2}-K^{2}\right) d V+\int_{M}\left(\nabla_{j} \rho_{i}-\frac{1}{n} \Delta \rho g_{j i}\right)\left(\nabla^{j} \rho^{2}-\frac{1}{n} \Delta \rho g^{j i}\right) d V=0
$$

Thus under the assumptions of the theorem, we can conclude

$$
\nabla_{j} \rho_{i}-\frac{1}{n} \Delta \rho \cdot g_{j i}=0
$$

which proves the theorem.

We also prove

THEOREM 5.2. If a complete Riemannian manifold $M$ of dimension $n>2$ admits an infinitesimal non-homothetic conformal transformation $v^{h}$ such that

$$
\mathcal{L} G_{j i}=0,
$$

then $M$ is isometric to a sphere.

Proof. From (2.13) and the assumption of the theorem, we have

$$
\nabla_{j} \rho_{i}-\frac{1}{n} \Delta \rho g_{j i}=0
$$

and consequently Theorem S of Tashiro proves the theorem.

\section{§6. Generalizations of Theorems $\mathrm{J}, \mathrm{K}$ and $\mathrm{L}$.}

We prove

Theorem 6.1. If a compact Riemannian manifold $M$ of dimension $n>2$ and with $K \neq 0$ admits an infinitesimal non-homothetic conformal transformation $v^{h}$ such that 


$$
\mathcal{L} K=0
$$

and

$$
\mathcal{L}\left\{\sum_{k=0}^{l}(-n+1)^{k} \alpha_{k} \tilde{\Delta}^{k}\left(\mathcal{L} f-\frac{n-2}{n+2} \mathcal{L} \Delta K\right)+\sum_{k=0}^{m}(-n+1)^{k} \beta_{k} \tilde{\Delta}^{k}\left(\mathcal{L} g-\frac{4}{n+2} \mathcal{L} \Delta K\right)\right\}=0,
$$

where $l$ and $m$ are non-negative integers, $\alpha_{k}$ and $\beta_{k}$ constants such that $\sum_{k=0}^{l} \alpha_{k}$ and $\sum_{k=0}^{m} \beta_{k}$ are non-negative and not both zero, and

$$
\begin{aligned}
\tilde{\Delta} F & =\Delta\left(\frac{1}{K} F\right), \\
\tilde{\Delta}^{2} F & =\Delta\left(\frac{1}{K} \Delta\left(\frac{1}{K} F\right)\right),
\end{aligned}
$$

for a scalar function $F$, then $M$ is isometric to a sphere.

Proof. When $\mathcal{L} K=0$, we have from (2.12)

$$
\rho=-\frac{n-1}{K} \Delta \rho
$$

and consequently

$$
\int_{M} \rho F d V=-(n-1) \int_{M} \frac{1}{K} F \Delta \rho d V=-(n-1) \int_{M} \rho \Delta\left(\frac{1}{K} F\right) d V
$$

for a scalar function $F$, or

$$
\begin{aligned}
\int_{M} \rho F d V= & (-n+1) \int_{M} \rho \tilde{\Delta} F d V \\
= & (-n+1)^{2} \int_{M} \rho \tilde{\Delta}^{2} F d V \\
& \cdots \cdots \cdots \cdots \cdots \cdots \\
= & (-n+1)^{k} \int_{M} \rho \tilde{\Delta}^{k} F d V .
\end{aligned}
$$

From (3. 4), we have

$$
\begin{aligned}
\int_{M}\left[\frac{2}{n-2} f \rho^{2}+\right. & \left.\frac{1}{2(n-2)} \rho\left(\mathcal{L} f-\frac{n-2}{n+2} \mathcal{L} \Delta K\right)\right] d V \\
& +\int_{M}\left(\nabla_{j} \rho_{i}-\frac{1}{n} \Delta \rho g_{j i}\right)\left(\nabla^{j} \rho^{i}-\frac{1}{n} \Delta \rho g^{j i}\right) d V=0
\end{aligned}
$$




$$
\begin{array}{r}
\int_{M}\left[\frac{2}{n-2} f \rho^{2}+\frac{(-n+1)}{2(n-2)} \rho \tilde{\Delta}\left(\mathcal{L} f-\frac{n-2}{n+2} \mathcal{L} \Delta K\right)\right] d V \\
\quad+\int_{M}\left(\nabla_{j} \rho_{i}-\frac{1}{n} \Delta \rho g_{j i}\right)\left(\nabla^{\jmath} \rho^{i}-\frac{1}{n} \Delta \rho g^{j i}\right) d V=0, \\
\ldots \ldots \ldots \ldots \ldots \ldots \ldots, \\
\int_{M}\left[\frac{2}{n-2} f \rho^{2}+\frac{(-n+1)^{l}}{2(n-2)} \rho \tilde{\Delta}_{l}\left(\mathcal{L} f-\frac{n-2}{n+2} \mathcal{L} \Delta K\right)\right] d V \\
+\int_{M}\left(\nabla_{j} \rho_{i}-\frac{1}{n} \Delta \rho g_{j i}\right)\left(\nabla^{j} \rho^{i}-\frac{1}{n} \Delta \rho g^{j i}\right) d V=0,
\end{array}
$$

by virtue of (6.1).

Thus multiplying these equations by $2(n-2) \alpha_{0}, 2(n-2) \alpha_{1}, \cdots, 2(n-2) \alpha_{l}$ respectively and adding all the equations thus obtained, we find

$$
\begin{aligned}
\int_{M}\left[4\left(\sum_{k=0}^{l} \alpha_{k}\right) f \rho^{2}\right. & \left.+\rho \sum_{k=0}^{l}(-n+1)^{k} \alpha_{k} \tilde{\Delta}^{k}\left(\mathcal{L} f-\frac{n-2}{n+2} \mathcal{L} \Delta K\right)\right] d V \\
& +2(n-2)\left(\sum_{k=0}^{l} \alpha_{k}\right) \int_{M}\left(\nabla_{j} \rho_{i}-\frac{1}{n} \Delta \rho g_{j i}\right)\left(\nabla^{\jmath} \rho^{i}-\frac{1}{n} \Delta \rho g^{j i}\right) d V=0 .
\end{aligned}
$$

We also have from (3.14)

$$
\begin{aligned}
& \int_{M}\left[\frac{1}{2} g \rho^{2}+\frac{1}{8} \rho\left(\mathcal{L} g-\frac{4}{n+2} \mathcal{L} \Delta K\right)\right] d V \\
& +\int_{M}\left(\nabla_{j} \rho_{i}-\frac{1}{n} \Delta \rho g_{j i}\right)\left(\nabla^{j} \rho^{i}-\frac{1}{n} \Delta \rho g^{j i}\right) d V=0, \\
& \int_{M}\left[\frac{1}{2} g \rho^{2}+\frac{1}{8}(-n+1) \rho \tilde{\Delta}\left(\mathcal{L} g-\frac{4}{n+2} \mathcal{L} \Delta K\right)\right] d V \\
& +\int_{M}\left(\nabla_{j} \rho_{i}-\frac{1}{n} \Delta \rho g_{j i}\right)\left(\nabla^{j} \rho^{2}-\frac{1}{n} \Delta \rho g^{j i}\right) d V=0, \\
& \int_{M}\left[\frac{1}{2} g \rho^{2}+\frac{1}{8}(-n+1)^{m} \rho \tilde{\Delta}^{m}\left(\mathcal{L} g-\frac{4}{n+2} \mathcal{L} \Delta K\right)\right] d V \\
& +\int_{M}\left(\nabla_{j} \rho_{i}-\frac{1}{n} \Delta \rho g_{j i}\right)\left(\nabla^{j} \rho^{i}-\frac{1}{n} \Delta \rho g^{j i}\right) d V=0,
\end{aligned}
$$

by virtue of (6.1).

Thus multiplying these equations by $8 \beta_{0}, 8 \beta_{1}, \cdots, 8 \beta_{m}$ respectively and adding all the equations thus obtained, we find 


$$
\begin{aligned}
\int_{M}\left[4\left(\sum_{k=0}^{m} \beta_{k}\right)\right. & \left.g \rho^{2}+\rho \sum_{k=0}^{m}(-n+1)^{k} \beta_{k} \tilde{\Delta}^{k}\left(\mathcal{L} g-\frac{4}{n+2} \mathcal{L} \Delta K\right)\right] d V \\
& +8\left(\sum_{k=0}^{m} \beta_{k}\right) \int_{M}\left(\nabla_{j} \rho_{i}-\frac{1}{n} \Delta \rho g_{j i}\right)\left(\nabla^{j} \rho^{i}-\frac{1}{n} \Delta \rho g^{j i}\right) d V=0 .
\end{aligned}
$$

Adding (6.2) and (6.3), we obtain

$$
\begin{array}{r}
\int_{M}\left[4\left(\sum_{k=0}^{l} \alpha_{k}\right) f \rho^{2}+4\left(\sum_{k=0}^{m} \beta_{k}\right) g \rho^{2}+\rho\left\{\sum_{k=0}^{l}(-n+1)^{k} \alpha_{k} \tilde{\Delta}^{k}\left(\mathcal{L} \rho-\frac{n-2}{n+2} \mathcal{L} \Delta K\right)\right.\right. \\
\left.\left.+\sum_{k=0}^{m}(-n+1)^{k} \beta_{k} \tilde{\Delta}^{k}\left(\mathcal{L} g-\frac{4}{n+2} \mathcal{L} \Delta K\right)\right\}\right] d V \\
+\left[2(n-2) \sum_{k=0}^{l} \alpha_{k}+8 \sum_{k=0}^{m} \beta_{k}\right] \int_{M}\left(\nabla_{j} \rho_{i}-\frac{1}{n} \Delta \rho g_{j i}\right)\left(\nabla^{\jmath} \rho^{2}-\frac{1}{n} \Delta \rho g^{j i}\right) d V=0
\end{array}
$$

Thus under the assumptions of the theorem, we have from (6.4)

$$
\nabla_{j} \rho_{i}-\frac{1}{n} \Delta \rho g_{j i}=0
$$

which proves the theorem by virtue of Theorem $\mathrm{S}$ of Tashiro.

We next prove

THEOREM 6.2. Suppose that a compact Riemannian manifold $M$ of dimension $n>2$ and with $K \neq 0$ satisfies

$$
\alpha_{0} f-\alpha_{1} \Delta\left(\frac{f}{K}\right)+\beta_{0} g-\beta_{1} \Delta\left(\frac{g}{K}\right)=\text { const. }
$$

where $\alpha_{0}, \alpha_{1}, \beta_{0}, \beta_{1}$ are non-negative constants not all zero such that if $n>6$

$$
\frac{\alpha_{1}}{n-1} \geqq \frac{n-6}{8} \alpha_{0} \geqq 0, \quad \frac{\beta_{1}}{n-1} \geqq \frac{n-6}{8} \beta_{0} \geqq 0 .
$$

If $M$ admits an infinitesimal non-homothetic conformal transformation $v^{h}$ such that

$$
\mathcal{L} K=0, \quad \mathcal{L} \Delta K=0,
$$

then $M$ is isometric to a sphere.

Proof. Since $\mathcal{L} K=0$, we have from (2.12)

$$
\rho=-(n-1) \frac{1}{K} \Delta \rho
$$

Thus we have, for an arbitrary function $F$, 


$$
\begin{aligned}
\int_{M} \rho \mathcal{L} F d V & =-(n-1) \int_{M} \Delta \rho \frac{1}{K} \mathcal{L} F d V \\
& =-(n-1) \int_{M} \Delta \rho \mathcal{L}\left(\frac{F}{K}\right) d V \\
& =-(n-1) \int_{M} \rho \Delta \mathcal{L}\left(\frac{F}{K}\right) d V
\end{aligned}
$$

by virtue of $\mathcal{L} K=0$.

Thus, taking account of (2.7), we have

$$
\begin{aligned}
\int_{M} \rho \mathcal{L} F d V & =-(n-1) \int_{M} \rho\left[\mathcal{L} \Delta\left(\frac{F}{K}\right)+2 \rho \Delta\left(\frac{F}{K}\right)-(n-2) \rho^{i} V_{i}\left(\frac{F}{K}\right)\right] d V \\
& =-(n-1) \int_{M}\left[\rho \mathcal{L} \Delta\left(\frac{F}{K}\right)+2 \rho^{2} \Delta\left(\frac{F}{K}\right)+\frac{1}{2}(n-2) \rho^{2} \Delta\left(\frac{F}{K}\right)\right] d V
\end{aligned}
$$

and consequently

$$
\int_{M} \rho \mathcal{L} F d V=-(n-1) \int_{M} \rho \mathcal{L} \Delta\left(\frac{F}{K}\right) d V-\frac{(n-1)(n+2)}{2} \int_{M} \rho^{2} \Delta\left(\frac{F}{K}\right) d V .
$$

Now substituting (2.7) into (3.2), that is,

$$
\int_{M}\left[G_{j i} \rho^{j} \rho^{i}+\frac{1}{2 n} \rho^{2} \Delta K\right] d V+\int_{M}\left(\nabla_{j} \rho_{i}-\frac{1}{n} \Delta \rho g_{j i}\right)\left(\nabla^{j} \rho^{2}-\frac{1}{n} \Delta \rho g^{j i}\right) d V=0,
$$

we find

$$
\int_{M}\left[2 \rho^{2} f+\frac{1}{2} \rho \mathcal{L} f+\frac{n-2}{4} \rho^{2} \Delta K\right] d V
$$

$$
+(n-2) \int_{M}\left(\nabla_{j} \rho_{i}-\frac{1}{n} \Delta \rho g_{j i}\right)\left(\nabla^{\jmath} \rho^{i}-\frac{1}{n} \Delta \rho g^{j i}\right) d V=0 .
$$

Substituting (6.8) in which $F=f$ into (6.9), we find

$$
\begin{gathered}
\int_{M}\left[2 \rho^{2} f-\frac{n-1}{2} \rho \mathcal{L} \Delta\left(\frac{f}{K}\right)-\frac{(n-1)(n-2)}{4} \rho^{2} \Delta\left(\frac{f}{K}\right)+\frac{n-2}{4} \rho^{2} \Delta K\right] d V \\
+(n-2) \int_{M}\left(\nabla_{j} \rho_{i}-\frac{1}{n} \Delta \rho g_{j i}\right)\left(\nabla^{j} \rho^{i}-\frac{1}{n} \Delta \rho g^{j i}\right) d V=0 .
\end{gathered}
$$

Substituting (2.20) into (3.2), we find

$$
\int_{M}\left[2 \rho^{2} g+\frac{1}{2} \rho \mathcal{L} g+\rho^{2} \Delta K\right] d V+4 \int_{M}\left(\nabla_{j} \rho_{i}-\frac{1}{n} \Delta \rho g_{j i}\right)\left(\nabla^{j} \rho^{i}-\frac{1}{n} \Delta \rho g^{j i}\right) d V=0
$$

Substituting (6.8) in which $F=g$ into (6.11), we find 


$$
\begin{aligned}
\int_{M}\left[2 \rho^{2} g-\frac{n-1}{2} \rho \mathcal{L} \Delta\left(-\frac{g}{K}\right)\right. & \left.-\frac{(n-1)(n-2)}{4} \rho^{2} \Delta\left(\frac{g}{K}\right)+\rho^{2} \Delta K\right] d V \\
& +4 \int_{M}\left(\nabla_{j} \rho_{i}-\frac{1}{n} \Delta \rho g_{j i}\right)\left(\nabla^{j} \rho^{i}-\frac{1}{n} \Delta \rho g^{j i}\right) d V=0 .
\end{aligned}
$$

Thus multiplying (6.9) by $\alpha_{0},(6.10)$ by $\alpha_{1} /(n-1),(6.11)$ by $\beta_{0},(6.12)$ by $\beta_{1} /(n-1)$ and adding the equations obtained, we find

$$
\begin{gathered}
\int_{M}\left[2\left(\alpha_{0}+\frac{\alpha_{1}}{n-1}\right) \rho^{2} f+2\left(\beta_{0}+\frac{\beta_{1}}{n-1}\right) \rho^{2} g+\frac{1}{2} \rho \mathcal{L}\left(\alpha_{0} f-\alpha_{1} \Delta\left(\frac{f}{K}\right)+\beta_{0} g-\beta_{1} \Delta\left(\frac{g}{K}\right)\right)\right. \\
\text { (6. 13) } \left.-\frac{n+2}{4} \rho^{2}\left\{\alpha_{1} \Delta\left(\frac{f}{K}\right)+\beta_{1} \Delta\left(\frac{g}{K}\right)\right\}+\left\{\frac{n-2}{4}\left(\alpha_{0}+\frac{\alpha_{1}}{n-1}\right)+\left(\beta_{0}+\frac{\beta_{1}}{n-1}\right)\right\} \rho^{2} \Delta K\right] d V \\
+\left\{(n-2)\left(\alpha_{0}+\frac{\alpha_{1}}{n-1}\right)+4\left(\beta_{0}+\frac{\beta_{1}}{n-1}\right)\right\} \int_{M}\left(\nabla_{j} \rho_{i}-\frac{1}{n} \Delta \rho g_{j i}\right)\left(\nabla^{j} \rho^{i}-\frac{1}{n} \Delta \rho g^{j i}\right) d V=0 .
\end{gathered}
$$

According to the assumption, we put

$$
\alpha_{0} f-\alpha_{1} \Delta\left(\frac{f}{K}\right)+\beta_{0} g-\beta_{1} \Delta\left(\frac{g}{K}\right)=c \text { (const.) }
$$

from which

$$
\alpha_{1} \Delta\left(\frac{f}{K}\right)+\beta_{1} \Delta\left(\frac{g}{K}\right)=\alpha_{0} f+\beta_{0} g-c
$$

and consequently $(6.13)$ becomes

$$
\begin{aligned}
& \int_{M}\left[\left(\frac{2 \alpha_{1}}{n-1}-\frac{n-6}{4} \alpha_{0}\right) \rho^{2} f+\left(\frac{2 \beta_{1}}{n-1}-\frac{n-6}{4} \beta_{0}\right) \rho^{2} g+\frac{n+2}{4} c \rho^{2}\right. \\
& \left.+\left\{\frac{n-2}{4}\left(\alpha_{0}+\frac{\alpha_{1}}{n-1}\right)+\left(\beta_{0}+\frac{\beta_{1}}{n-1}\right)\right\} \rho^{2} \Delta K\right] d V \\
& +\left\{(n-2)\left(\alpha_{0}+\frac{\alpha_{1}}{n-1}\right)+4\left(\beta_{0}+\frac{\beta_{1}}{n-1}\right)\right\} \int_{M}\left(\nabla_{j} \rho_{i}-\frac{1}{n} \Delta \rho g_{j i}\right)\left(\nabla^{j} \rho^{2}-\frac{1}{n} \Delta \rho g^{j i}\right) d V=0 .
\end{aligned}
$$

On the other hand, from (6.14), we have by integration,

$$
0=\int_{M}\left(\alpha_{0} f+\beta_{0} g\right) d V-c \int_{M} d V
$$

which shows that

$$
c \geqq 0 \text {. }
$$

Also we have from (2.9) 


$$
\int_{M} \rho^{2} \Delta K d V=\frac{2}{n+1} \int_{M} \rho[\Delta(\mathcal{L} K)-\mathcal{L}(\Delta K)] d V=0
$$

and consequently equation (6.15) gives

$$
\nabla_{j} \rho_{i}-\frac{1}{n} \Delta \rho g_{j i}=0
$$

which proves the theorem.

We next prove

THEOREM 6. 3. If a compact Riemannian manifold $M$ of dimension $n>2$ with $K>0$ admits an infinitesimal non-homothetic conformal transformation $v^{h}$ such that

$$
\mathcal{L} K=0, \quad \mathcal{L} \Delta K=0,
$$

$$
\mathcal{L} \mathcal{L}\left\{\alpha_{0} f+\alpha_{1} \Delta\left(\frac{f}{K}\right)+\beta_{0} g+\beta_{1} \Delta\left(\frac{g}{K}\right)\right\}=0
$$

$\alpha_{0}, \alpha_{1}, \beta_{0}, \beta_{1}$ being constants not all zero such that

$$
4(n-1) \alpha_{0} \geqq(n+6) \alpha_{1} \geqq 0, \quad 4(n-1) \beta_{0} \geqq(n+6) \beta_{1} \geqq 0,
$$

then $M$ is isometric to a sphere.

Proof. We have from (6.8)

(6. 19) $\int_{M} \rho \mathcal{L} f d V=-(n-1) \int_{M} \rho \mathcal{L} \Delta\left(\frac{f}{K}\right) d V-\frac{(n-1)(n+2)}{2} \int_{M} \rho^{2} \Delta\left(\frac{f}{K}\right) d V$.

On the other hand, we have

$$
\begin{aligned}
\int_{M} \rho^{2} \Delta\left(\frac{f}{K}\right) d V & =\int_{M} \Delta \rho^{2} \frac{f}{K} d V=2 \int_{M}\left(\rho \Delta \rho+\rho_{i} \rho^{i}\right) \frac{f}{K} d V \\
& =\int_{M}\left(-\frac{2 K}{n-1} \rho^{2}+2 \rho_{i} \rho^{2}\right) \frac{f}{K} d V
\end{aligned}
$$

and consequently (6.19) becomes

(6. 20)

$$
\int_{M} \rho \mathcal{L} f d V=-(n-1) \int_{M} \rho \mathcal{L} \Delta\left(\frac{f}{K}\right) d V+(n+2) \int_{M} \rho^{2} f d V-(n-1)(n+2) \int_{M} \rho_{i} \rho^{i} \frac{f}{K} d V .
$$

Similarly we have 
296

KENTARO YANO AND SUMIO SAWAKI

$$
\begin{aligned}
\int_{M} \rho \mathcal{L} g d V=- & (n-1) \int_{M} \rho \mathcal{L} \Delta\left(\frac{g}{K}\right) d V \\
& +(n+2) \int_{M} \rho^{2} g d V-(n-1)(n+2) \int_{M} \rho_{i} \rho^{i} \frac{g}{K} d V .
\end{aligned}
$$

Now, from (6.9), we have

$$
\begin{aligned}
\int_{M}\left[\frac{2}{n-2} \rho^{2} f+\right. & \left.\frac{1}{2(n-1)} \rho \mathcal{L} f+\frac{1}{4} \rho^{2} \Delta K\right] d V \\
& +\int_{M}\left(\nabla_{j} \rho_{i}-\frac{1}{n} \Delta \rho g_{j i}\right)\left(\nabla^{j} \rho^{2}-\frac{1}{n} \Delta \rho g^{j i}\right) d V=0 .
\end{aligned}
$$

Substituting (6.20) into (6.21), we obtain

$$
\begin{gathered}
\int_{M}\left[\frac{n+6}{2(n-2)} \rho^{2} f-\frac{n-1}{2(n-2)} \rho \mathcal{L} \Delta\left(\frac{f}{K}\right)-\frac{(n-1)(n+2)}{2(n-2)} \rho_{i} \rho^{2} \frac{f}{K}+\frac{1}{4} \rho^{2} \Delta K\right] d V \\
+\int_{M}\left(\nabla_{j} \rho_{i}-\frac{1}{n} \Delta \rho g_{j i}\right)\left(\nabla^{j} \rho^{2}-\frac{1}{n} \Delta \rho g^{j i}\right) d V=0 .
\end{gathered}
$$

From (6.11) we have

$$
\begin{aligned}
\int_{M}\left[\frac{1}{2} \rho^{2} g+\frac{1}{8} \mathcal{L}\right. & \left.+\frac{1}{4} \rho^{2} \Delta K\right] d V \\
& +\int_{M}\left(\nabla_{j} \rho_{i}-\frac{1}{n} \Delta \rho g_{j i}\right)\left(\nabla^{j} \rho^{i}-\frac{1}{n} \Delta \rho g^{j i}\right) d V=0 .
\end{aligned}
$$

Substituting (6.21) into (6.24), we obtain

$$
\begin{aligned}
& \int_{M}\left[\frac{n+6}{8} \rho^{2} g\right.\left.-\frac{n-1}{8} \rho \mathcal{L} \Delta\left(\frac{g}{K}\right)-\frac{(n-1)(n+2)}{8} \rho_{i} \rho^{2} \frac{g}{K}+\frac{1}{4} \rho^{2} \Delta K\right] d V \\
&+\int_{M}\left(\nabla_{j} \rho_{i}-\frac{1}{n} \Delta \rho g_{j i}\right)\left(\nabla^{j} \rho^{2}-\frac{1}{n} \Delta \rho g^{j i}\right) d V=0 .
\end{aligned}
$$

Multiplying (6.22) by $\alpha_{0}$, (6.23) by $-\alpha_{1} /(n-1),(6.24)$ by $4 \beta_{0} /(n-2),(6.25)$ by $-4 \beta_{1} /(n-1)(n-2)$ and adding the results thus obtained, we find

$$
\begin{aligned}
& \int_{M}\left[\left(\frac{2 \alpha_{0}}{n-2}-\frac{n+6}{2(n-1)(n-2)} \alpha_{1}\right) \rho^{2} f+\left(\frac{2 \beta_{0}}{n-2}-\frac{n+6}{2(n-1)(n-2)} \beta_{1}\right) \rho^{2} g\right. \\
& \quad+\frac{1}{2(n-2)} \rho \mathcal{L}\left(\alpha_{0} f+\alpha_{1} \Delta\left(\frac{f}{K}\right)+\beta_{0} g+\beta_{1} \Delta\left(\frac{g}{K}\right)\right)+\frac{n+2}{2(n-2)}\left(\alpha_{1} f+\beta_{1} g\right) \frac{1}{K} \rho_{i} \rho^{2} \\
& \left.\quad+\frac{1}{4}\left(\alpha_{0}-\frac{1}{n-1} \alpha_{1}+\frac{1}{n-2} \beta_{0}-\frac{4}{(n-1)(n-2)} \beta_{1}\right) \rho^{2} \Delta K\right] d V \\
& +\frac{1}{4}\left(\alpha_{0}-\frac{1}{n-1} \alpha_{1}+\frac{4}{n-2} \beta_{0}-\frac{4}{(n-1)(n-2)} \beta_{1}\right) \int_{M}\left(\nabla_{j} \rho_{i}-\frac{1}{n} \Delta \rho g_{j i}\right)\left(\nabla^{j} \rho^{i}-\frac{1}{n} \Delta \rho g^{j i}\right) d V=0 .
\end{aligned}
$$


We have here

$$
\int_{M} \rho^{2} \Delta K d V=0
$$

by virtue of (2.9), $\mathcal{L} K=0$ and $\mathcal{L} \Delta K=0$, and

$$
\begin{aligned}
& \alpha_{0}-\frac{1}{n-1} \alpha_{1} \geqq \frac{n+6}{4(n-1)} \alpha_{1}-\frac{1}{n-1} \alpha_{1}=\frac{n+2}{4(n-1)} \alpha_{1} \geqq 0, \\
& \beta_{0}-\frac{1}{n-1} \beta_{1} \geqq \frac{n-6}{4(n-1)} \beta_{1}-\frac{1}{n-1} \beta_{1}=\frac{n+2}{4(n-1)} \beta_{1} \geqq 0,
\end{aligned}
$$

$\alpha_{0}, \alpha_{1}, \beta_{0}, \beta_{1}$ not being zero at the same time, and consequently

$$
\alpha_{0}-\frac{1}{n-1} \alpha_{1}+\frac{4}{n-2} \beta_{0}-\frac{4}{(n-1)(n-2)} \beta_{1} \geqq 0,
$$

thus (6.26) gives

$$
\nabla_{j} \rho_{i}-\frac{1}{n} \Delta \rho g_{j i}=0
$$

which proves the theorem.

\section{$\S 7$. Generalizations of Theorems $I, N, 0, Q$ and $R$.}

We prove

THEOREM 7.1. Suppose that $M$ is a compact and orientable Riemannian manifold of dimension $n>2$ and with scalar curvature $K$ and admits an infinitesimal non-homothetic conformal transformation $v^{h}$ such that $\mathcal{L} K=0$. Then in order for $M$ to be isometric to a sphere, it is necessary and sufficient that

$$
\int_{M}\left(G_{j i} \rho^{j} \rho^{2}+\frac{1}{2 n} \rho^{2} \Delta K\right) d V=0
$$

Proof. Since the necessity is evident, we prove the sufficiency. When $\mathcal{L} K=0$, we have from (3.2)

$$
\int_{M}\left(G_{j i} \rho^{j} \rho^{i}+\frac{1}{2 n} \rho^{2} \Delta K\right) d V+\int_{M}\left(\nabla_{j} \rho_{i}-\frac{1}{n} \Delta \rho g_{j i}\right)\left(\nabla^{j} \rho^{i}-\frac{1}{n} \Delta \rho g^{j i}\right) d V=0 .
$$

Thus, from the assumption, we can conclude that

$$
\nabla_{j} \rho_{i}-\frac{1}{n} \Delta \rho g_{j i}=0,
$$

which proves the theorem. 
Theorem 7.2. If $M$ is a complete Riemannian manifold of dimension $n \geqq 2$ with scalar curvature $K$ and admits an infinitesimal non-homothetic conformal transformation $v^{h}: \mathcal{L} g_{j i}=2 \rho g_{j i}, \rho \neq$ const. such that $\mathcal{L} K=0$, then

$$
\rho^{2} K^{2} \leqq n(n-1)^{2}\left(\nabla_{j} \rho_{i}\right)\left(\nabla^{j} \rho^{i}\right),
$$

equality holding if and only if $M$ is isometric to a sphere.

Proof. We have

$$
\left(\nabla_{j} \rho_{i}-\frac{1}{n} \Delta \rho g_{j i}\right)\left(\nabla^{j} \rho^{2}-\frac{1}{n} \Delta \rho g^{j i}\right)=\left(\nabla_{j} \rho_{i}\right)\left(\nabla^{j} \rho^{i}\right)-\frac{1}{n}(\Delta \rho)^{2} \geqq 0,
$$

from which

$$
\frac{1}{n}(\Delta \rho)^{2} \leqq\left(\nabla_{j} \rho_{i}\right)\left(\nabla^{j} \rho^{i}\right)
$$

But since $\mathcal{L} K=0$, we have from (2.12)

$$
\Delta \rho=-\frac{1}{n-1} K \rho,
$$

and consequently

$$
\rho^{2} K^{2} \leqq n(n-1)^{2}\left(\nabla_{j} \rho_{i}\right)\left(\nabla^{j} \rho^{i}\right),
$$

equality holding if and only if $M$ is isometric to a sphere.

THEOREM 7.3. If $M$ is a compact and orientable Riemannian manifold of dimension $n>2$ with the scalar curvature $K$ and admits an infinitesimal nonhomothetic conformal transformation $v^{h}: \mathcal{L} g_{j i}=2 \rho g_{j i}, \rho \neq$ const. such that $\mathcal{L} K=0$, then

$$
n(n-1) \int_{M} K_{j i} \rho^{j} \rho^{i} d V \leqq \int_{M} \rho^{2} K^{2} d V
$$

equality holding if and only if $M$ is isometric to a sphere.

Proof. Since $\mathcal{L} K=0$, we have

$$
\Delta \rho=-\frac{1}{n-1} \rho K
$$

and consequently, we have, from (2.4),

$$
\int_{M}\left[K_{j i} \rho^{j} \rho^{i}-\frac{1}{n(n-1)} \rho^{2} K^{2}\right] d V+\int_{M}\left(\nabla_{j} \rho_{i}-\frac{1}{n} \Delta \rho g_{j i}\right)\left(\nabla^{j} \rho^{2}-\frac{1}{n} \Delta \rho g^{j i}\right) d V=0 \text {, }
$$

from which 


$$
n(n-1) \int_{M} K_{j i} \rho^{j} \rho^{i} d V \leqq \int_{M} \rho^{2} K^{2} d V
$$

equality holding if and only if

$$
\nabla_{j} \rho_{i}-\frac{1}{n} \Delta \rho g_{j i}=0
$$

that is, $M$ is isometric to a sphere.

Theorem 7.4. If $M$ is a compact Riemannian manifold of dimension $n>2$ and admits an infinitesimal non-homothetic conformal transformation such that

$$
\mathcal{L} K=0, \quad \mathcal{L} \mathcal{L}\left(G_{j i} G^{j i}\right)=0 \quad \mathcal{L} \mathcal{L} \Delta K=0,
$$

or

$$
\mathcal{L} K=0, \quad \mathcal{L} \mathcal{L}\left(Z_{k j i h} Z^{k j i \hbar}\right)=0, \quad \mathcal{L} \mathcal{L} \Delta K=0,
$$

then $M$ is isometric to a sphere.

Proof. The first part of the theorem is a special case of Corollary 1 to Theorem 3. 1 and the second part is a special case of Theorem 3.2.

\section{BIBLIOGRAPHY}

[1] Goldberg, S. I., Manifolds admitting a one-parameter group of conformal transformations. Michigan Math. J. 15 (1968), 339-344.

[2] Goldberg, S. I., AND S. Kobayashi, The conformal transformation group of a compact Riemannian manifold. Amer. J. Math. 84 (1962), 170-174.

[3] Hsiung, C. C., On the group of conformal transformations of a compact Riemannian manifold. Proc. Nat. Acad. Sc1. U.S.A. 54 (1965), 1509-1513.

[4] Ishinara, S., On infinitesimal concircular transformations. Kōdai Math. Sem. Rep. 12 (1960), 45-56.

[5] Ishinara, S., AND Y. TASHiro, On Riemannian manifolds admitting a concircular transformation. Math. J. Okayama Univ. 9 (1959), 19-47.

[6] Lichnerowicz, A., Sur les transformations conformes d'une variété riemannienne compacte. C. R. Acad. Sc1. Paris 259 (1964), 697-700.

[7] Obata, M., Certan conditions for a Riemannian manifoll to be isometric with a sphere. J. Math. Soc. Japan 14 (1962), 333-340.

[8] Oвата, M., Conformal transformations in Riemannian manifolds (in Japanese). Sūgaku, Math. Soc. Japan 14 (1963), 152-164.

[9] Oвата, M., Riemannian manifolds admitting a solution of a certain system of differential equations. Proc. U.S.-Japan Seminar in Differential Geometry, Kyōto, Japan (1965), 101-114.

[10] Овата, M., Quelques inégalités intégrales sur une variété riemannienne compacte. C. R. Acad. Sci. Paris 264 (1967), 123-125.

[11] Tashiro, Y., Complete Riemannian manifolds and some vector fields. Trans. Amer. Math. Soc. 117 (1965), 251-275. 
[12] Weber, W. C., AND S. I. Goldberg, Conformal deformations of Riemannian manifolds. Queen's Papers on Pure and Applied Mathematics, Queen's University, Kingston, Canada (1969).

[13] YaNo, K., On harmonic and Killing vector fields. Annals of Math. 55 (1952), $38-45$.

[14] YANO, K., The theory of Lie derivatives and its applications. North Holland Publ. Co., Amsterdam (1957).

[15] YANO, K., On Riemannian manifolds with constant scalar curvature admitting a one-parameter conformal transformation group. Proc. Nat. Acad. Sci. U.S.A. 55 (1966), 472-476.

[16] YANO, K., Riemannian manifolds admitting a conformal transformation group. Proc. Nat. Acad. Sc1. U.S.A. 62 (1969), 314-319.

[17] YANO, K., On Riemannian manifolds admitting an infinitesimal conformal transformation. Math. Zeitschr. 113 (1970), 205-214.

[18] YaNO, K., AND T. NAGANO, Einstein spaces admittıng a one-parameter group of conformal transformations. Annals of Math. 69 (1959), 451-461.

[19] Yano, K., AND M. Obata, Sur le groupe de transformations conformes dans une variété de Riemann dont le scalaire de courbure est constant. C. R. Acad. Sci. Paris 260 (1965), 2698-2700.

[20] YANO, K., AND S. SAWAKI, On Riemannian manifolds admitting a conformal transformation group. J. of Diff. Geom. 2 (1968), 161-184.

Tokyo Institute of Technoloy And

Nigata University. 